\title{
A importância da doação de leite humano na contribuição do desenvolvimento aos
}

\section{recém-nascidos prematuros}

\author{
The importance of human milk donation in the contribution of development to premature
}

newborns

La importancia de la donación de leche materna en la contribución al desarrollo de los recién nacidos prematuros

Recebido: 14/05/2021 | Revisado: 27/05/2021 | Aceito: 01/06/2021 | Publicado: 17/06/2021

\author{
Gabriela Bucci de Souza \\ ORCID: https://orcid.org/0000-0002-5931-5416 \\ Universidade Estácio de Sá, Brasil \\ gabibucci01@gmail.com \\ Dayana Campos de Oliveira Cardoso \\ ORCID: https://orcid.org/0000-0002-5031-6041 \\ Universidade Estácio de Sá, Brasil \\ camposdayana2015@ hotmail.com \\ Camila Vieira Correia \\ ORCID: https://orcid.org/0000-0003-2352-8134 \\ Universidade Estácio de Sá, Brasil \\ camila.uol@hotmail.com \\ Mariane Marques dos Santos Bongestad \\ ORCID: https://orcid.org/0000-0002-7097-0497 \\ Universidade Estácio de Sá, Brasil \\ gersonemariane@gmail.com \\ Priscila Cristina Pereira de Oliveira da Silva \\ ORCID: https://orcid.org/0000-0002-6304-7952 \\ Universidade Estácio de Sá, Brasil \\ prioliveira0512@gmail.com
}

\begin{abstract}
Resumo
Introdução: O banco de leite humano visa desenvolver uma reflexão sobre a necessidade da doação de leite humano para os recém-nascidos prematuros, a fim de promover o alimento primordial, de proteção e desenvolvimento para sua vida. Objetivo: Descrever o caminho para a doação de leite materno ainda no pré-natal e informar a importância da educação em saúde abordando o aleitamento materno e o percurso para doação. Metodologia: Trata-se de uma pesquisa de revisão integrativa, com abordagem qualitativa de natureza básica e objetivo exploratório, que teve com base dados a Biblioteca Virtual de Saúde Enfermagem usando os Descritores em Ciências da Saúde (DeCS): Banco de leite; Nutrição materna; Doação de leite. Após as buscas com os descritores foram selecionados artigos completos, em língua portuguesa, publicados entre 2016 a 2021.Diante dos critérios obtidos, alcançamos 13 artigos que versaram sobre o tema proposto para construção do estudo. Resultados: O estudo mostra -se dados obtidos que apontou a necessidade da segurança e do beneficiamento do LH, dados no qual, deverão ser transcritos com achados encontrados, a fim de alcançar o objetivo desse estudo. Considerações Finais: É indispensável atuação do enfermeiro, sendo de extrema importância para a criação do vínculo frente as dificuldades enfrentadas pelas nutrizes de conhecer o banco de leite e incentivar seu uso, visando a educação em saúde dessas mulheres e o crescimento profissional do enfermeiro diante do tema.
\end{abstract}

Palavras-chave: Banco de leite; Nutrição materna; Doação de leite.

\begin{abstract}
Introduction: The human milk bank aims to develop a reflection on the need to donate human milk to premature newborns, in order to promote the primordial, protective and developmental food for their life. Objective: To describe the way to donate breast milk while in prenatal care and to inform the importance of health education addressing breastfeeding and the route for donation. Methodology: This is an integrative review research, with a qualitative approach of a basic nature and exploratory objective, which was based on data from the Virtual Health Library Nursing using the Health Sciences Descriptors (DeCS): Milk bank; Maternal nutrition; Donation of milk. After searching with the descriptors, full articles were selected, in Portuguese, published between 2016 and 2021. Based on the criteria obtained, we reached 13 articles that dealt with the proposed theme for the construction of the study.Results: The study shows data obtained that pointed out the need for safety and improvement of LH, data in which, they must be transcribed with found findings, in order to reach the objective of this study. Final
\end{abstract}


Considerations: Nurses' performance is essential, being extremely important for the creation of a bond in view of the difficulties faced by nursing mothers to know the milk bank and encourage its use, aiming at the health education of these women and the professional growth of nurses in the face of theme.

Keywords: Milk bank; Maternal nutrition; Donation of milk.

\section{Resumen}

Introducción: El banco de leche materna tiene como objetivo desarrollar una reflexión sobre la necesidad de donar leche materna a los recién nacidos prematuros, con el fin de promover el alimento primordial, de protección y desarrollo para su vida. Objetivo: Describir la forma de donar leche materna durante el prenatal e informar sobre la importancia de la educación en salud que aborde la lactancia materna y la ruta de donación. Metodología: Se trata de una investigación de revisión integradora, con abordaje cualitativo de carácter básico y objetivo exploratorio, que se basó en datos de la Biblioteca Virtual en Salud Enfermería utilizando los Descriptores de Ciencias de la Salud (DeCS): Banco de Leite; Nutrición materna; Donación de Leche. Luego de buscar con los descriptores, se seleccionaron los artículos completos, en portugués, publicados entre 2016 y 2021. Con base en los criterios obtenidos, llegamos a 13 artículos que abordaron el tema propuesto para la construcción del estudio. Resultados: El estudio muestra los datos obtenidos. que señaló la necesidad de seguridad y mejora de la LH, datos en los cuales, se deben transcribir con los hallazgos encontrados, para alcanzar el objetivo de este estudio. Consideraciones finales: El desempeño del enfermero es fundamental, siendo de suma importancia para la creación de un vínculo ante las dificultades que enfrentan las madres lactantes para conocer el banco de leche y fomentar su uso, con el objetivo de la educación en salud de estas mujeres y el crecimiento profesional de las mismas. enfermeras frente al tema.

Palabras clave: Banco de leche; Nutrición materna; Donación de leche.

\section{Introdução}

É fundamental uma boa nutrição para a promoção do crescimento normal e desenvolvimento integral do recémnascido. O neonato a termo e de alto risco apresentam muitas limitações para que se possam ser atendidas as necessidades básicas para a promoção do crescimento. (MS, 2015)

A classificação da idade gestacional dos recém-nascidos pré-termo (RNPT) são aqueles nascidos antes da $37^{\mathrm{a}}$ semana de gestação. Já os recém-nascidos a termo (RNT) são aqueles nascidos no intervalo entre o começo do primeiro dia, da $37^{\mathrm{a}}$ semana de gestação e o fïm do último dia, da 42a semana de gestação. (Sassá et al. 2014)

Amamentar é além de nutrir a criança. É um exercício que engloba interação entre mãe e filho, com repercussões no estado nutricional da criança, em sua destreza de se defender contra infecções, no seu desenvolvimento cognitivo e emocional, em sua fisiologia e em sua saúde ao longo do tempo, além de ter implicações na saúde física e psíquica da mãe. (MS, 2015)

É importante conhecer e empregar as definições de aleitamento materno adotadas pela Organização Mundial de Saúde e reconhecidas no mundo inteiro. Assim, o aleitamento materno costuma ser classificado em: aleitamento materno exclusivo quando a criança recebe somente o leite da mãe ou leite humano de outra fonte; aleitamento materno predominante - quando recebe além do leite materno, água, sucos da fruta; aleitamento materno complementado - quando além do leite materno, qualquer alimento semissólido ou sólido com o objetivo de complementá-lo e não de substituí-lo; aleitamento materno quando recebe o leite direto materno (ordenhado ou direto da mama e aleitamento materno misto ou parcial - quando recebe leite materno e outros tipos de leite. (MS, 2015)

A alimentação de bebês a termos e neonatos prematuros é fundamental para melhorar a sobrevivência infantil e promover um crescimento e desenvolvimento saudável. Segundo à OMS recomenda-se o aleitamento materno por dois anos ou mais, sendo exclusivo até 6 meses, além de apresentar benefícios a longo prazo ao Recém-nascido, garante recuperação ao estado materno. (MS, 2017)

O aleitamento materno propicia uma nutrição de alta qualidade para a criança, a concentração de gordura no leite aumenta ao decorrer de uma mamada, colaborando com o ganho de peso do bebê, mantendo a produção suficiente para atendêlo. Também contribui com inúmeros fatores imunológicos que o protege, sendo a forma mais sensível, natural e econômica para a redução da morbilidade infantil. (MS, 2015) 
Segundo Hochenberry, (2018) O Crescimento e desenvolvimento manifestam o somatório de incontáveis mudanças que ocorrem no decorrer da vida do indivíduo. O Crescimento resulta no aumento de tamanho e peso, no todo ou em alguma parte e desenvolvimento no surgimento e amplificação das capacidades do indivíduo por meio do crescimento, maturação e aprendizado.

Estima-se outras possíveis vantagens para as mulheres entre o aleitamento materno e a redução da prevalência do câncer de mama; evita nova gravidez, e menores custos financeiros com gastos de fórmulas infantis, materiais artificiais, com uso de mamadeira e bicos, além de promover qualidade de vida e fortalecimento familiar. (MS, 2011)

O Brasil é citado como exemplo de país com mais políticas de proteção e promoção ao aleitamento materno, amplo leque com ações iniciativas e estratégicas. Cabe ressaltar em 1998, houve expansão da criação da Rede Brasileira de Banco de Leite Humano, que desempenham papel importante, bem como missão de promover, proteger e apoiar o aleitamento materno, orientar e distribuir leite humano com qualidade, prioritariamente aos Recém-Nascidos e de baixo peso, que não sugam, infectados, dentre outros. (MS, 2017)

A Rede de Banco de Leite Humano é considerada a maior e mais complexa do mundo pela Organização Mundial de Saúde (WHO). É responsável pela promoção do aleitamento materno e cumprimento das atividades de coleta, processamento e monitorização da qualidade do leite produzido nos primeiros dias após o parto (colostro), leite de transição e leite maduro. (Fiocruz, 2011)

O colostro, que é o leite secretado pela mãe nos primeiros 7 dias após o parto, é de extrema importância para o crescimento saudável do neonato, já que ele é rico em microorganismos, principalmente bactérias que habitam no corpo humano. Muitas puérperas produzem um volume excessivo de leite em seu pós parto, além da necessidade do seu bebê, com isso se tornam possíveis doadoras de leite materno, desde que, sejam saudáveis e estejam com exames negativados para transmissão de doenças e infecções que são transmitidas através do leite materno e ainda não são eliminados no processo de pasteurização feitos para comprovar na hora da doação. (MS, 2018)

O leite vindo de doações, é muito utilizado em UTI (Unidade de Terapia Intensiva), e unidades Intermediárias (UI), seu uso tem sido muito incentivado, podendo ser ofertados tanto o leite extraído diretamente do seio materno e oferecido imediatamente ao recém-nascido, quando proveniente de banco de leite ordenhado, visto que, ajuda a garantir uma adequada evolução pôndero-estatural, na recuperação e formação dos prematuros. Edualeide et al. (2011)

Diante do exposto, o presente estudo tem como objeto de estudo: dificuldades e fatores limitantes as nutrizes no processo de doação do leite humano. E, portanto, temos o seguinte questionamento: quais as dificuldades encontradas pelas nutrizes para se tornarem uma doadora do banco de leite humano?

O objetivo geral do estudo é: analisar as dificuldades e fatores limitantes as nutrizes para a doação de leite humano na intenção de conhecer essas mulheres, afim de direcionar ações educativas e de promoção ao aleitamento materno, colaborando com o aumento do número de doadoras resultando em um maior volume de leite coletado.

E como objetivos específicos: descrever o caminho para a doação do leite materno ainda no pré-natal pois é nesse período que a mãe começa a se preparar para a lactação e está sensível ao aprendizado e analisar a importância da Educação em Saúde, abordando o aleitamento materno e o percurso para a doação do leite ordenhado.

Além disso, o estudo se dá pela importância da equipe de saúde está preparada e treinada, pois estes deverão contribuir para a sensibilização da doação do leite materno, que melhora a qualidade de vida de bebês prematuros internados em unidade de terapia intensiva, impossibilitados de se alimentar ao seio materno.

Vale salientar, que o presente estudo pretende contribuir para os enfermeiros, em seu trabalho, pois é de suma importância o incentivo e a influência de prática promotoras de cuidado, sobre a doação o alimento materno e a motivar essas nutrizes sadias, a se tornarem doadoras de leite materno humano. 


\section{Metodologia}

Trata-se de uma pesquisa do tipo revisão integrativa de abordagem qualitativa, onde será trabalhada a importância da doação de leite para os bancos especializados.

Ao ingressar no sistema de ensino superior se torna indispensável o planejamento e práticas de trabalhos de pesquisas e extensão. Efetivamente, as iniciações científicas poderiam ser executadas por todo corpo social com intuito de se organizar para as novas técnicas e modificações que acontecem no decorrer dos anos. Pereira et al. (2018).

Bardin (2016), define que a pesquisa qualitativa é a que se fundamenta principalmente em análises qualitativas, caracterizando-se, em princípio, pela não utilização de instrumental estatístico na análise dos dados.

Segundo Ganong (1987) a revisão integrativa da literatura propõe o estabelecimento de critérios bem definidos sobre a coleta de dados, análise e apresentação dos resultados, desde o início do estudo, a partir de um protocolo de pesquisa previamente elaborado e validado.

Para a construção do artigo abordou-se a revisão integrativa da literatura, que consiste na elaboração de análise abrangente de artigos, estabelecendo critérios de investigação técnico-científico para coletar dados, analisar e apresentar resultados. As informações obtidas podem sinalizar achados que requerem mais atenção no meio científico, visando melhorias na prática profissional (Ganong, 1987)

A revisão integrativa necessita ser guiada por etapas. De acordo com Mendes, Silveira e Galvão (2008) essa revisão deverá conter seis etapas: 1) Estabelecimento de hipótese ou questão de pesquisa; 2) Amostragem ou busca na literatura; 3) Categorização dos estudos; 4) Avaliação dos estudos incluídos na revisão; 5) Interpretação dos resultados; 6) Síntese do conhecimento ou apresentação da revisão.

A estratégia de identificação e seleção dos artigos originou-se através da busca da Biblioteca Virtual de Saúde (BVS), no mês de março de 2021. Iniciou-se a busca de materiais que contivessem em seu resumo os descritores em ciências da saúde (DeCS) "Banco de leite"; "nutrição materna"; "doação de leite" com uso do boleador AND. Foram adotados os seguintes critérios para seleção dos artigos: materiais completos, em língua portuguesa, no formato de artigo e com recorte temporal de dez anos (2011-2021). Os critérios de exclusão foram os estudos que não atendessem aos critérios citados anteriormente e duplicados.

Emergiram 6 artigos com os critérios citados, os quais procederam-se à leitura minuciosa para a construção do conteúdo, destacando aqueles que responderam ao objetivo proposto pelo estudo, com intuito de organizar os dados.

Os artigos foram tabelados por título, autores, ano de publicação, periódico, objetivos, resultados e conclusão. A partir de então iniciou a análise bibliométrica destes que foram agrupados por similaridade sob forma de categorias.

\section{Resultados e Discussão}

Os resultados apresentados no estudo mostram as evidências levantadas com a leitura dos artigos previamente selecionados. 
Quadro 1: Características dos artigos analisados no período de 2011 a 2021.

\begin{tabular}{|c|c|c|c|c|c|c|}
\hline Título & Autoria & $\begin{array}{l}\text { Base de } \\
\text { dados }\end{array}$ & Ano & Objetivo & Métodos & Considerações Finais \\
\hline $\begin{array}{l}\text { O papel do banco } \\
\text { de leite humano na } \\
\text { promoção da saúde } \\
\text { materno infantil }\end{array}$ & $\begin{array}{l}\text { Rafaela Mara; } \\
\text { Luana } \\
\text { Cupertino; } \\
\text { Sylvia do } \\
\text { Carmo; } \\
\text { Bruno David }\end{array}$ & MEDLINE & 2021 & $\begin{array}{l}\text { Detectar o papel do } \\
\text { BLH na promoção } \\
\text { da saúde materno } \\
\text { infantil. }\end{array}$ & $\begin{array}{c}\text { Revisão } \\
\text { sistemática }\end{array}$ & $\begin{array}{l}\text { Identificou-se que as } \\
\text { atividades } \\
\text { desenvolvidas pelo } \\
\text { BLH foram positivas } \\
\text { na promoção do } \\
\text { aleitamento materno. }\end{array}$ \\
\hline $\begin{array}{l}\text { O papel do } \\
\text { Obstetra no } \\
\text { incentivo ao } \\
\text { aleitamento } \\
\text { materno }\end{array}$ & $\begin{array}{c}\text { Mariani Neto; } \\
\text { Myiashita }\end{array}$ & $\begin{array}{l}\text { Sec. Est. } \\
\text { Saúde SP }\end{array}$ & 2018 & $\begin{array}{c}\text { Orientar as mães o } \\
\text { valor da } \\
\text { amamentação } \\
\text { exclusiva até o } 6 \\
\text { meses e } \\
\text { suplementar até os } \\
2 \text { anos. Quanto ao } \\
\text { leite excedente, a } \\
\text { possibilidade de } \\
\text { doação ao BLH. }\end{array}$ & Monografia & $\begin{array}{l}\text { Verificou-se que o } \\
\text { Obstetra tem grande } \\
\text { importância ao } \\
\text { incentivo ao } \\
\text { aleitamento materno e } \\
\text { possível doação. }\end{array}$ \\
\hline $\begin{array}{c}\text { Fatores que } \\
\text { interferem na } \\
\text { doação de leite } \\
\text { humano }\end{array}$ & $\begin{array}{l}\text { Flávia Pinhão; } \\
\text { Daiani Oliveira; } \\
\text { Cristiane } \\
\text { Cardoso; } \\
\text { Stela Maris; }\end{array}$ & LILACS & 2016 & $\begin{array}{c}\text { Verificar os fatores } \\
\text { que limitam a } \\
\text { doação de leite. }\end{array}$ & $\begin{array}{c}\text { Revisão } \\
\text { integrativa }\end{array}$ & $\begin{array}{l}\text { Os profissionais da } \\
\text { saúde possuem } \\
\text { influência no estímulo } \\
\text { à doação de leite. }\end{array}$ \\
\hline $\begin{array}{l}\text { Doação de leite } \\
\text { humano: } \\
\text { dificuldades e } \\
\text { fatores limitantes }\end{array}$ & $\begin{array}{l}\text { Larissa Santos; } \\
\text { Maria Vanessa; } \\
\text { Maria José; } \\
\text { Mônica } \\
\text { Santiago }\end{array}$ & LILACS & 2011 & $\begin{array}{c}\text { Reconhecer os } \\
\text { impasses para a } \\
\text { doação de leite } \\
\text { humano do } \\
\text { Hospital Leonor } \\
\text { Mendes de Barros }\end{array}$ & $\begin{array}{c}\text { Estudo de } \\
\text { prevalência }\end{array}$ & $\begin{array}{l}\text { Através de entrevista } \\
\text { realizada com as } \\
\text { pacientes, a maioria } \\
\text { informou que doaria o } \\
\text { leite excedente. }\end{array}$ \\
\hline $\begin{array}{l}\text { Banco de leite } \\
\text { humano na } \\
\text { perspectiva da } \\
\text { mulher doadora }\end{array}$ & $\begin{array}{c}\text { Valdecyr Herdy; } \\
\text { Diego Pereira; } \\
\text { Maria Bertilla; } \\
\text { Rosangela de } \\
\text { Matos; } \\
\text { Renata Rangel; } \\
\text { Flavia do Valle }\end{array}$ & BDENF & 2013 & $\begin{array}{c}\text { Entender o } \\
\text { pensamento a das } \\
\text { nutrizes mediante a } \\
\text { ação de doar. }\end{array}$ & $\begin{array}{c}\text { Estudo } \\
\text { qualitativo }\end{array}$ & $\begin{array}{l}\text { As nutrizes confiam } \\
\text { que a doação de leite } \\
\text { transforma o mundo. }\end{array}$ \\
\hline $\begin{array}{l}\text { Caracterização de } \\
\text { nutrizes doadoras } \\
\text { de um banco de } \\
\text { leite humano }\end{array}$ & $\begin{array}{c}\text { Mariana de } \\
\text { Oliveira; } \\
\text { Bibiane Dias; } \\
\text { Flavia } \\
\text { Aparecida; } \\
\text { Nara dos Santos; } \\
\text { Juliana Cristina; } \\
\text { Flávia Gomes }\end{array}$ & BDENF & 2013 & $\begin{array}{c}\text { Analisar o } \\
\text { entendimento sobre } \\
\text { o processo de } \\
\text { doação. }\end{array}$ & $\begin{array}{c}\text { Estudo de } \\
\text { prevalência }\end{array}$ & $\begin{array}{c}\text { Poucas mulheres } \\
\text { receberam } \\
\text { informações sobre a } \\
\text { doação durante o pré- } \\
\text { natal. }\end{array}$ \\
\hline
\end{tabular}

Fonte: Autores.

Após análise dos artigos selecionados neste estudo foram construídas as seguintes categorias: 
Categoria I: Aleitamento materno e seus benefícios.

O leite humano é a melhor opção de nutrição, por seu valor nutricional, bem como imunológico, para o recémnascido. Além dos benefícios nutricionais e imunológicos, que estão disponíveis em qualidades e quantidades que os tornam facilmente digeríveis pelo intestino do neonato e absorvidos para o desenvolvimento e crescimento, também traz benefícios psicológicos para o binômio mãe-filho, fortalecendo o elo afetivo. (Hochenberry, 2018)

Segundo Iniciativa Hospital Amigo da Criança (IHAC), o Brasil adota medidas de fortalecimento ao aleitamento materno, para encorajar, promover e apoiar a amamentação, como modelo para o seu sucesso de melhor nutrição para os recém-nascidos.

A amamentação do neonato, é um fator importante para o correto desenvolvimento e crescimento craniofacial em quesito ósseo, muscular e funcional, pois mantém estruturas prontas para exercerem o desenvolvimento da musculatura orofacial. Por esse motivo, a amamentação materna é uma forma de prevenção a problemas futuros da criança, pois proporciona um preparo da condição neuromuscular das estruturas da boca, por isso, não é aconselhável o uso de bicos de silicone, chupetas para tentar facilitar a lactação, pois afeta toda a formação futura. (MS, 2017)

O ingurgitamento mamário, se desenvolve por congestão, retenção de leite nos alvéolos e também por edema advindo da obstrução do sistema linfático, originado pela alta demanda da produção de leite, sendo incompatível com a necessidade do bebê. Quando isso ocorre, as mamas ficam completamente distendidas, podendo gerar dor e febre, sendo assim dificulta a amamentação do neonato, o que agrava mais o quadro desta puérpera. Nesses casos é de extrema importância a ordenha manual para não acumular e ingurgitar, dando a opção da mãe realizar a doação desse leite, a menos que não tenha evoluído para uma mastite e esteja com secreção purulenta. (MS, 2011)

O período da amamentação dura em média dois anos e o desmame ocorre de forma natural pelo bebê ou induzida pela própria mãe, de forma que sempre seja respeitado o tempo da criança, para que não haja possíveis traumas futuros. Com o aleitamento materno exclusivo até os seis meses, não há necessidade de complementação de frutas, sucos, chás entre outros, pois não traz nenhuma vantagem fisiológica, podendo até gerar prejuízos à saúde. (MS, 2015)

É notório que muitas mulheres possuem o medo de amamentar nos primeiros meses de vida do recém nato, muitas não recebem as devidas orientações como por exemplo, expor as mamas ao sol de 15 a 20 minutos por dia, não passar pomadas, óleos, nenhuma solução, pois pode fazer queimaduras ou deixar as mamas mais sensíveis, devem passar o próprio leite em toda aréola para diminuir o risco de fissuras e mais pra frente o risco de mastite, que pode gerar pus, edema e dor. (MS, 2015)

Categoria II: O papel do banco de leite humano na promoção da saúde aos prematuros.

A Rede Brasileira de Bancos de Leite Humano, é considerada, pela OMS, a maior e mais complexa do mundo, consiste em uma unidade especializada ligada a unidade de cuidados intensivos prestados ao neonato e seu principal objetivo é estimular a doação de leite materno para alimentar recém-nascidos hospitalizados em risco, cujas mães, por fatores diversos, não estão aptas a amamentar. (Fiocruz, 2020)

Essas unidades são responsáveis pela pasteurização e proteção do aleitamento materno, coletar e distribuir leite humano certificada, a fim de suprir as necessidades do público alvo diminuindo os índices de morbimortalidade infantil, bem como cumprir com o que é preconizado pela Academia de Pediatria e pela OMS, visando a melhoria da qualidade de vida da população. (Fiocruz, 2020)

Vale salientar que o Banco de Leite Humano (BLH) é um estabelecimento sem fins lucrativos, sendo proibida a compra e venda de seu produto - leite humano - que deve ser doado por nutrizes em lactação, garantindo que a doação seja 
exclusivamente do excedente, já que sabe-se que o leite materno é suficiente para satisfazer todas as necessidades nutricionais de todos os tipos de prematuros. (Santos DT et al., 2010)

'’O Banco de Leite Humano é um centro especializado, responsável pela promoção e o incentivo ao aleitamento materno e execução de atividades de coleta, processamento e controle de qualidade do colostro, leite de transição e leite humano maduro, para posterior distribuição, sob prescrição de médicos ou de nutricionistas, sendo este obrigatoriamente vinculado a um hospital materno e ou infantil. E uma instituição sem fins lucrativos, sendo vedada a comercialização dos produtos por ela distribuídos"'. Maia et al (2006)

Uma das prioridades dos BLHs no Brasil é de atender as mães de recém-nascidos pré-termo e de baixo peso internados em unidade de terapia intensiva. É importante lembrar que 1 litro de leite materno doado pode alimentar até 10 recém-nascidos por dia, além disso dependendo do peso do prematuro $1 \mathrm{ml}$ é suficiente para nutrí-lo. (Fiocruz, 2011)

Contudo, existem fatores históricos, socioeconômicos, culturais e individuais que podem interferir na produção de leite materno e na amamentação, entre outros. Porém sabe-se que por dificuldades diversas, como falta de informações, uso de medicamentos, incompatíveis para doação, doenças como vírus da imunodeficiência humana (HIV), entre outros, fazendo com que seja necessário recorrer a outros métodos para alcançar um ganho ponderal apropriado, neste contexto, destacam-se os Bancos de Leite Humano. (MS, 2015)

Categoria III:O processo de doação do leite materno aos Bancos de Leite

O primeiro banco de leite humano do Brasil foi implantado em outubro de 1943 no então Instituto Nacional de Puericultura, atual Instituto Fernandes Figueira (IFF). O seu principal objetivo era coletar e distribuir leite humano (LH) visando em atender os casos a exemplo prematuridade, distúrbios metabólicos, além da função imunológica comprometida. Contudo, foi com o desenvolvimento do Programa Nacional de Incentivo ao Aleitamento Materno (PNIAM), a partir de 1985 que os BLHs passaram assumir um novo papel no cenário da saúde pública brasileira, adotando pontos estratégicos para as ações de promoção, proteção e apoio à amamentação. Maia et al, (2006)

Esse novo modelo ocorre em um período de franca expansão e resulta de um processo histórico em parceria entre área da criança do Ministério da Saúde e a Fundação Oswaldo Cruz, projeto esse que viabilizou ações de desenvolvimento tecnológico, criando opções de baixo custo, centrados no processamento e no controle de qualidade do leite humano. (MS, 2015)

O leite humano era distribuído de forma cru, sem receber qualquer tipo de tratamento de qualidade, contudo, em decorrência do grande volume de leite coletado fez-se necessário a esterilização de mamadeiras, em banho-maria por 20 minutos. As doadoras eram em sua totalidade pobres, medidas que encontravam na comercialização do leite e nas demais benesses uma forma de sustento, não havia a preocupação de resgatar a lactação das mães com estímulo à amamentação. (Maia et al. (2006)

Há consenso de que os resultados positivos alcançados pela RBLH difere por ter uma atuação voltada para o incentivo à amamentação e também por trabalhar o leite humano ordenhado na perspectiva dos referenciais da tecnologia do alimento ofertando aos bebês incapazes de se alimentarem. Maia et al. (2006)

O banco de leite é um serviço especializado obrigatoriamente vinculado a um hospital de atenção materno/infantil, responsáveis por ações de promoção, proteção e apoio ao aleitamento materno e execução das demandas de coleta e classificação da produção lática da nutriz, processamento e controle de qualidade de colostro, leite de transição e leite humano maduro e distribuição, sendo proibido a comercialização dos seus produtos distribuídos. Tatiana et al. (2017) 
O Posto de Coleta de Leite Humano (PCLH) é uma unidade fixa ou móvel, intra ou extra hospitalar vinculado ao banco de leite humano, responsável pela execução de atividades de coleta da produção lática da nutriz, e sua estocagem, além disso, da promoção, proteção e apoio ao aleitamento materno, porém as questões do processamento do leite, são exclusivas ao banco de leite humano. (Fiocruz, 2020)

Existem atualmente 17 Bancos de Leite Humano e 7 Postos de Coleta implantados no Estado do Rio de Janeiro (Fiocruz, 2020). Segundo o Ministério da Saúde, as doadoras de leite humano são nutrizes sadias que apresentam produção láctea superior às exigências do seu filho, que se dispõem a doar por livre e espontânea vontade o excesso de leite produzido. (MS, 2018)

Em estudo realizado por Maia et al. (2006), a triagem das doadoras deve ser realizada por um profissional treinado e capacitado, no momento do primeiro contato com a nutriz com o BLH ou PCLH, mediante o preenchimento do formulário de cadastro que obtenha as seguintes informações: dados pessoais; ser saudável; estar amamentando;apresentar exames do prénatal; não usar drogas ilícitas ou álcool; realizar exames (hemograma completo, VDRL, Anti-HIV) e demais sorologias, quando não apresentar o cartão do pré-natal ou quando a nutriz não tiver feito o pré-natal e não usar medicamentos incompatíveis com a amamentação.

O leite materno quando coletado pela mãe, deverá ser armazenado em um freezer ou congelador, bem tampado e com identificação com nome, data e hora da coleta. Sempre deixando um espaço de aproximadamente dois dedos da tampa. É imprescindível que no momento da coleta a mulher lave bem as mãos com sabão, lavar as mamas também mas somente com água limpa; usar touca e máscara para evitar contaminação do leite. O frasco deverá ser de vidro e de preferência, que seja transparente com tampa plástica; a validade do leite materno nos frascos é de 12 horas quando guardado na geladeira e de 15 dias quando armazenado no freezer. (MS, 2018)

Para alimentar o bebê é necessário o uso de copinhos ou até mesmo de seringa para facilitar a sua livre mamada, no momento do preparo desse leite é recomendado que seja feito em banho-maria, mornando o leite aos poucos. Quando aquecido o suficiente, agitar lentamente para misturar os componentes do leite, que se separam quando o frasco está em repouso. (MS, 2018)

\section{Conclusão}

A partir do exposto, percebe-se que o investimento na infraestrutura torna-se indispensável, assim como a construção de programas especializados, para que seja um local capaz de ofertar total amparo e conforto para as doadoras, mantendo o acompanhamento e o cuidado, tornando-se um vínculo, pois é notório que a demanda ainda se mantém alta pela procura de leite materno para recém natos em Unidades de Terapia Intensiva, que dependem deste gesto para sobreviver.

É possível concluir neste estudo que os principais fatores para uma boa execução no sistema do Banco de Leite Humano, é o aprimoramento na atenção primária, divulgando e abordando as mulheres no pré-natal, intervindo de maneira satisfatória e efetiva, enfatizando os benefícios para ambos os lados, para que se tornem possíveis doadoras, além de prevenir o ingurgitamento mamário e infecções secundárias, sendo valorizadas com dignidade e humanidade em sua posição de doadora.

A pesquisa exibe como condição limitadora a escassez de estudos nessa área. Aguarda-se que este estudo promova a prática de novas investigações que exponham todos os benefícios relacionados ao tema exibido e possibilite a análise das equipes de saúde com a intenção de estimular e potencializar essa atividade para os centros de saúde do Brasil. 
Research, Society and Development, v. 10, n. 7, e15210716095, 2021

(CC BY 4.0) | ISSN 2525-3409 | DOI: http://dx.doi.org/10.33448/rsd-v10i7.16095

\section{Referências}

Bardin, L. (2016). Análise de contéudo. Almedina Brasil.

Edualeide, J.P.B.N., Rosineide, S.B. (2011). Envolvimento de pais com a dieta oferecida ao filho prematuro: relato de experiência. Revista de Enfermagem Ufpe, 5(5), 1302-1305.

Fiocruz. (2020). Rede Brasileira de Bancos de Leite Humano.

Fiocruz. (2020). Higiene e condutas: doadoras.

Ganong, L.H. (1987). Integrative reviews of nursin research. New Tork, 10(11), 1-11.

Hochenberry, M. J. (2018). Fundamentos de enfermagem pediátrica, Elsevier.

Maia, P. R., Almeida, J. A., Novak, F. R., \& Silva, D. A. (2006). Rede Nacional de Bancos de Leite Humano: gênese e evolução. Revista Brasileira de Saúde Materno Infantil, 6(3), 285-292.

Mendes, K., Silveira, R. C., \& Galvão, C. M. (2008). Revisão integrativa: metódo de pesquisa para a incorporação de evidências na saúde e na enfermagem. Texto Contexto Enfermagem, Florianopólis, 17(4): 758-64.

Ministério da saúde. (2011). Doação de leite materno beneficia bebês doentes e prematuros.

Ministério da saúde. (2018). Os dois lados da doação de leite materno.

Ministério da saúde. (2015). Saúde da criança: Aleitamento materno e alimentação complementar, Caderno de atenção básica.23 (2a ed).

Ministério da saúde. (2017). Bases para discussão da política nacional de promoção, proteção e apoio ao aleitamento materno. Livro 1, (23).

Pereira, A. S.; Shitsuka, D. M.; Parreira, F. J.; \& Shitsuka, R. (2018). Metodologia Da Pesquisa Científica. UFSM. https://repositorio.ufsm.br/bitstream/handle/ 1/15824/Lic_Computacao_Metodologia-Pesquisa-Cientifica.pdf?sequence=1

Sassá, A. H., Schmidt, K. T., Rodrigues, B. C., Ichisato, S. M., Higarashi, I. H., \& Marcon, S. S. (2014). Bebês pré-termo: aleitamento materno e evolução ponderal. Revista Brasileira de Enfermagem.

Tatiana, M. X. M., Maria, I. C. O., \& Cristiano, S. B. (2017). Prevalência à doação de leite para postos de recebimento de leite humano de unidades básicas de saúde, Revista de Pediatria, 93(4), 383-388. 